\title{
Stokes-Flow Problem Solved Using Maple
}

\author{
Pratibha ${ }^{1}$ and D.J. Jeffrey \\ Department of Applied Mathematics, \\ The University of Western Ontario, London, Ontario, Canada \\ ${ }^{1}$ Present address: Information Technology Development Agency (ITDA), \\ Government of Uttaranchal,272-B, Phase II, Vasant Vihar, \\ Dehradun, India 248006
}

\begin{abstract}
An unusual boundary-value problem that arises in a fluidmechanical application is solved to high precision, as a challenge problem in scientific computation. A second-order differential equation must be solved on $(0, \infty)$, subject to boundary conditions that specify only the asymptotic behaviour of the solution at the two ends of the solution domain. In addition, the solution is required to high accuracy to settle a conjecture made by previous authors. The solution is obtained by computing multiple series solutions using Maple.
\end{abstract}

\section{Introduction}

Computations of fluid flow sometimes lead to unusual problems in the solution of ordinary differential equations. The present problem comes from a paper by O'Neill and Stewartson [1. The problem posed is an excellent test case for the application of computer algebra systems, such as MAPLE, to scientific computation. A function $A(s)$ satisfies the differential equation

$$
s^{3} K^{\prime} A^{\prime \prime}+s A^{\prime}\left[s^{2} K^{\prime \prime}+3 s K^{\prime}+2 K\right]-A\left[s^{2} K^{\prime \prime}+4 s K^{\prime}+2 K\right]=-s^{2} X^{\prime \prime},
$$

where $K=s^{-1}-\operatorname{coth} s$ and $X=\operatorname{coth} s-1$, subject to the boundary conditions that $A(s)$ is no more singular than $s^{-2}$ at the origin and that $A(s)$ decays at infinity. The equation has a regular singular point at $s=0$ and an irregular point at infinity. In terms of this function, constants $k_{1}$ and $k_{2}$ must be calculated according to the formulae

$$
\begin{aligned}
k_{1}= & \frac{4}{5}+\frac{1}{2} \int_{0}^{\infty}\left\{\left(A+\frac{3}{5 s^{2}}\right)\left[2 s \operatorname{csch}^{2} s-(\operatorname{coth} s-1)\left(1+2 s+s^{2} \operatorname{csch}^{2} s\right)\right]\right. \\
& -\frac{3}{5} e^{-2 s}\left[\frac{1}{s^{3}}+\frac{1}{s^{2}}+\frac{2}{3 s}+\frac{5}{3}(2 s-1) \operatorname{coth} s\right] \\
& \left.-\frac{3}{5}\left[\frac{2}{s^{3}}-\left(\frac{3}{s^{2}}+\frac{2}{s}\right)(\operatorname{coth} s-1)\right]\right\} d s, \\
k_{2}= & \frac{1}{5}+\frac{1}{4} \int_{0}^{\infty}\left[4 s A+s^{2} A^{\prime \prime} K+2(\operatorname{coth} s-1)-4 e^{-2 s} / 5 s\right] d s .
\end{aligned}
$$


The challenge is to verify (by computation) that the two constants are equal.

The problem is an ideal one for exploring ways in which MAPLE can contribute to computational problems. Because of the singular points in the equation, the standard way to solve them numerically is to generate the first steps of a numerical solution using a series expansion about the origin, and about infinity. The truncation of the series and the change from one integration method to another generate some inaccuracies that might be acceptable in other contexts. However, since the question concerns the difference between the constants at the fifth and sixth significant figures, we need a highly accurate solution. Here we show that such a solution can be obtained by MAPLE with relatively little effort on the part of the user, the work being done by routines that we developed in 2 .

The use of series expansions in the solution of differential equations has always been hampered by several difficulties. The first difficulty is the laborious nature of their derivation. This is compounded by the second difficulty, which is the slow rate of convergence of the series. In order to obtain acceptable accuracy, many terms are needed, but the calculation of these is tedious. The final difficulty is their radius of convergence. Often a singularity in the complex plane will prevent the series converging at all points of interest, although the function is well behaved on the real line.

All of these difficulties can be overcome in this case using MAPLE. The new routines developed in 2] allow us to obtain large numbers of terms in the series with little effort and quickly. Using this fact we can re-expand the solution at different points along the axis, thus working around the convergence problem. We shall thus obtain a highly accurate solution and prove that the two constants $k_{1}$ and $k_{2}$ are indeed equal.

\section{Solutions About $s=0$}

The differential equation (11) has a regular singular point at the origin and another in the complex plane at $s=i \pi$. We denote the homogeneous solutions about $s=0$ as $A_{h}^{(0)}$ and the particular integral as $A_{p}^{(0)}$. Using MAPLE we found each as a series expression correct to 75 terms.

$$
A_{h}^{(0)}=s^{(-2+\sqrt{10})}\left[1+\sum_{\substack{n=2 \\ n \text { even }}}^{74} a_{n} s^{n}\right]
$$

where the first few constants in the series are

$$
a_{2}=\frac{13}{270}-\frac{2 \sqrt{10}}{135}, \quad a_{4}=-\frac{31}{2268}+\frac{967 \sqrt{10}}{226800}, \quad a_{6}=\frac{5}{6804}-\frac{313 \sqrt{10}}{1360800} .
$$

The particular integral $A_{p}^{(0)}$, correct to 75 terms is found as

$$
A_{p}^{(0)}=\sum_{\substack{n=-2 \\ n \text { even }}}^{74} b_{n} s^{n}
$$


where

$$
\begin{aligned}
b_{-2} & =-\frac{3}{5}, & b_{0}=-\frac{6}{25} \\
b_{2} & =-\frac{107}{1575}, & b_{4}=\frac{394}{307125}
\end{aligned}
$$

We reject the other homogeneous solution because asymptotically it behaves as $O\left(s^{-2-\sqrt{10}}\right)$, for small values of $s$. Hence for small $s$, the general solution of differential equation (1) is

$$
A(s)=A_{p}^{(0)}+c A_{h}^{(0)},
$$

where the constant $c$ has to be determined.

\section{Series Solutions About Other Expansion Points}

The solutions $A_{h}^{(0)}$ and $A_{p}^{(0)}$ converge only for $s<\pi$. This is unavoidable because of the singularity on the imaginary axis. We expand the functions $A_{p}^{(0)}$ and $A_{h}^{(0)}$ along the real axis by analytic or numerical continuation. We use the solutions $A_{h}^{(0)}$ and $A_{p}^{(0)}$ to give us boundary conditions at a new expansion point. We begin with the series solutions about $s=5 / 2$, an ordinary point of the differential equation (11). We find the homogeneous solution $A_{h}^{(5 / 2)}$ of the homogeneous version of the differential equation (1) (i.e. right-hand side is zero) subject to the boundary conditions

$$
A_{h}^{(5 / 2)}=\left.A_{h}^{(0)}\right|_{s=5 / 2}, \quad \frac{d}{d s} A_{h}^{(5 / 2)}=\left.\frac{d}{d s} A_{h}^{(0)}\right|_{s=5 / 2} .
$$

Similarly, we find the particular integral $A_{p}^{(5 / 2)}$. The general solution of the differential equation (1) about $s=5 / 2$ is

$$
A(s)=A_{p}^{(5 / 2)}+c A_{h}^{(5 / 2)} .
$$

Continuing in this way, we find the solutions at expansion points $s=4,6,8$ etc. To achieve high accuracy, all the solutions were found correct to 75 terms.

\section{Asymptotic Solution}

About $s=\infty$, we obtain an asymptotic expansion as follows.

For large $s, \operatorname{coth}(s) \sim 1$. We therefore replace coth $s$ with 1 and find the particular integral $a_{p}$ and the homogeneous solution $a_{h}$ for the resulting equation to be

$$
a_{h}=e^{-2 s}, \quad a_{p}=-2 s^{2} e^{-2 s} .
$$


We neglect the other homogeneous solution which is $1-2 s$ for large $s$.

Higher order approximations are found in the same manner. Hence for large $s$, the differential equation (10) has the asymptotic solution

$$
A(s) \sim a_{p}+C a_{h}
$$

where the constant $C$ has to be determined.

\section{The Constants $C$ and $c$}

We find the constants $C$ and $c$ by matching the two solutions, for $s$ small and for $s$ large. The two solutions and their derivatives are equal for those values of $s$ where these solutions converge. By solving the two equations, we get the constants $c$ and $C$ as given in Table (11).

Table 1. Values of constants $C$ and $c$ for different $N$

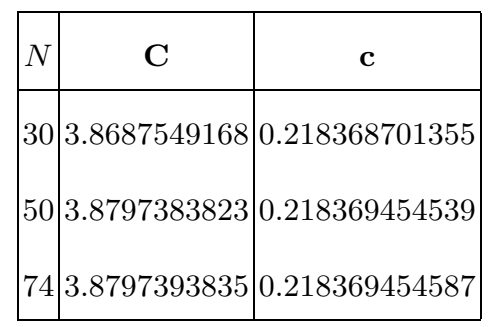

\section{$6 \quad$ Definite Integrals $k_{1}$ and $k_{2}$}

Since we have series expansions at all points, the evaluation of the integral is equally straightforward. The integrals $k_{1}$ and $k_{2}$ are found as

$$
\begin{aligned}
& k_{1}=-0.2622674637, \\
& k_{2}=-0.2622674637 .
\end{aligned}
$$

The programs used for the series solutions of ODEs and the programs used in this particular computation can be obtained from the authors.

\section{References}

1. M.E. O'Neill and K. Stewartson 1967 "On the slow motion of a sphere parallel to a nearby plane wall" J. Fluid Mech. 27, 705-724.

2. Pratibha 1995 "Maple tools for hydrodynamic interaction problems", PhD Thesis, The University of Western Ontario, London, Canada. 